\title{
High-speed dynamic atomic force microscopy by using a Q-controlled cantilever eigenmode as an actuator
}

\author{
M. Balantekin \\ Electrical and Electronics Engineering, İzmir Institute of Technology, Urla, İzmir 35430, Turkey
}

\section{A R T I C L E I N F O}

\section{Article history:}

Received 25 August 2013

Received in revised form

2 November 2014

Accepted 16 November 2014

Available online 20 November 2014

Keywords:

Atomic force microscope

High-speed dynamic AFM

Fast actuation

Q-controlled eigenmode of a cantilever

\begin{abstract}
A B S T R A C T
We present a high-speed operating method with feedback to be used in dynamic atomic force microscope (AFM) systems. In this method we do not use an actuator that has to be employed to move the tip or the sample as in conventional AFM setups. Instead, we utilize a Q-controlled eigenmode of an AFM cantilever to perform the function of the actuator. Simulations show that even with an ordinary tappingmode cantilever, imaging speed can be increased by about 2 orders of magnitude compared to conventional dynamic AFM imaging.
\end{abstract}

(c) 2014 Elsevier B.V. All rights reserved.

\section{Introduction}

Atomic Force Microscope (AFM) [1] has become a widely used and indispensable tool in nano-science and technology. The dynamic modes of AFM have surpassed the contact-mode imaging due to increased force sensitivity and reduced lateral forces. Although the commercial systems provide required spatial resolution for structural analysis, the imaging speed of dynamic AFM systems is not satisfactory for certain applications. It is important to minimize the error due to thermal and mechanical drift and the time spent for any nanoscale imaging experiment. Moreover, increasing the scan speed will indeed enable the use of dynamic AFM systems much effectively in industrial scale nanometrology.

The bandwidth of the actuator that adjusts the vertical position of the sample or the cantilever and the transient response of the cantilever are the two major factors that limit the scan speed. In addition, the $\mathrm{X}-\mathrm{Y}$ scanner, the electronic detector and the feedback controller have all effect on the scan speed [2-7]. To increase the imaging bandwidth, both the sensor and the actuator sizes have been minimized at the expense of increased system complexity [7-13]. Fast X-Y scanners have been developed [14-18]. Novel probe structures with integrated actuators were also designed [19-25]. Although non-dynamic methods without feedback have been proposed [26,27], these methods may not be suitable for delicate samples.

The dynamic-AFM systems can be divided into two main categories: the amplitude modulation (AM) AFM, also called

E-mail address: mujdatbalantekin@iyte.edu.tr tapping-mode, and the frequency modulation (FM) AFM, also called noncontact-mode [28]. The AFM systems operated in air usually utilizes AM modulation method due to its simplicity. The FM-AFM method is preferred in atomic resolution imaging in vacuum to increase the imaging bandwidth, but it can also be operated in air. The multi-frequency excitation of FM-AFM systems has lately gained attraction in fast force spectroscopy and material characterization at the atomic scale as well [29-31]. In this study, we propose a high-speed dynamic imaging method with feedback that can be employed in both AM-AFM and FM-AFM systems.

\section{Method}

The operating principle of the method is to adjust the oscillation amplitude of the $Q$-controlled fundamental eigenmode to keep the higher eigenmode oscillation amplitude (in AM-AFM) or frequency (in FM-AFM) at a constant level. Hence, the distance between the tip and the sample surface is adjusted by changing the oscillation amplitude of fundamental eigenmode of the cantilever instead of using an actuator as in conventional AFM systems. In other words, the higher eigenmode oscillation is used for sensing while the $Q$-controlled fundamental eigenmode oscillation is used for actuation. The $Q$-control enables the fundamental eigenmode oscillation amplitude reach the steady-state faster.

In Fig. 1, we explain the operation of the method. We defined an example sample surface and we plotted the total tip oscillation showing the motion of tip in different regions of the sample surface. During Phase I, the tip is in steady interaction with the 


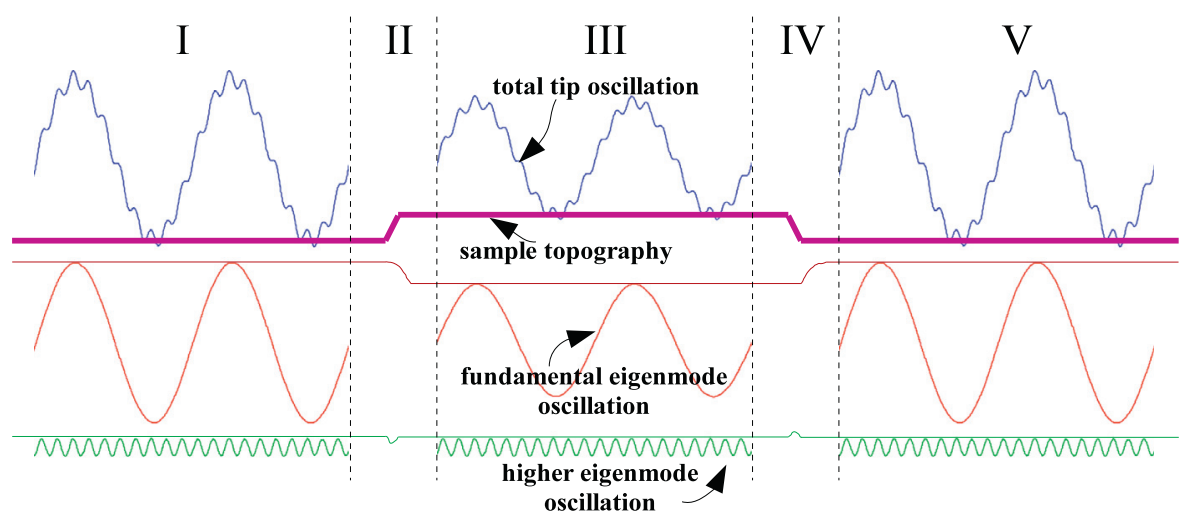

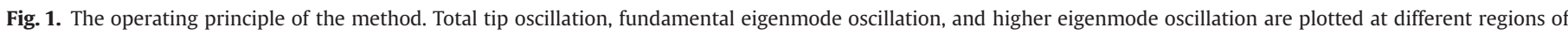
sample topography.

surface and the total tip oscillation has two components. The first component is fundamental eigenmode oscillation and the second component is higher eigenmode oscillation. The fundamental eigenmode oscillation has a much higher amplitude compared to higher eigenmode oscillation, and the feedback controller is trying to keep either the amplitude (in AM-AFM) or the frequency (in FM-AFM) of the higher eigenmode oscillation at a constant level. If we assume that the scan direction is from left to right, then, the tip will experience a raised surface feature in Phase II. This will change either the amplitude or the frequency of the higher eigenmode oscillation, as indicated by a small wiggle in the horizontal line drawn just above the higher eigenmode oscillation. This in turn is going to change the feedback controller output, and the feedback controller output will adjust the amplitude of the fundamental eigenmode oscillation accordingly. The decrease in amplitude, indicated by a line shown just above the fundamental eigenmode oscillation, must ideally be the inverse of the sample topography (Phase III). In Phase IV, the decrease in sample topography causes an opposite change in the higher eigenmode oscillation amplitude or frequency with respect to Phase II, which in turn causes an opposite change in the fundamental eigenmode oscillation amplitude. After the adjustment of fundamental eigenmode oscillation amplitude by the feedback controller the tip is again in steady interaction with the sample surface (Phase V).

It is known that the bulky Z-piezo of conventional AFM systems is the main factor limiting the system bandwidth and hence the scan speed due to its low resonant frequency. On the other hand, a typical tapping-mode cantilever has a fundamental resonant frequency on the order of a few hundred $\mathrm{kHz}$. Therefore we expect a faster transient response to the topographical variations when a cantilever used as an actuator. However, a typical tapping-mode cantilever also has a high $Q$-factor both in air and vacuum environments. The higher the $Q$-factor the higher the time required for fundamental eigenmode oscillation to reach the steady-state. We overcome this problem by applying the $Q$-control $[32,33]$ to reduce the artificial $Q$-factor of the fundamental eigenmode.

The point-mass model equations for fundamental and higher eigenmodes are given in Eqs. (1) and (2), respectively. $y_{1}(t)$ and $y_{n}$ $(t)$ are the tip oscillations, $F_{1}$ and $F_{n}$ are the driving forces, $w_{1}$ and $w_{n}$ are the free resonance frequencies, and $Q_{1}$ and $Q_{n}$ are the $Q-$ factors of the fundamental and higher eigenmodes, respectively. Mathematically, the time derivative of fundamental eigenmode oscillation $y_{1}(t)$ is multiplied with a constant gain $G_{\mathrm{Q}}$ and added to the velocity proportional term in Eq. (1). By properly selecting the value of $G_{Q}$ one can decrease the artificial $Q$-factor of the fundamental eigenmode. If there will be a change in the tip-sample interaction force $f_{T S}(t)$ while scanning the sample surface, this change is sensed by $y_{\mathrm{n}}(t)$, and the feedback controller will adjust the driving force of the fundamental eigenmode by multiplying $G_{\text {cont }}\left[y_{\mathrm{n}}(t)\right]$ with $F_{1} \cos \left(w_{1} t\right)$. This in turn will change the amplitude of $y_{1}(\mathrm{t})$. We expect that this response due to any variation in surface topography will be much faster than a response of a Z-piezo since the amplitude of $y_{1}(t)$ will reach the steady-state faster with $Q$-control.

$$
\begin{aligned}
& \frac{d^{2} y_{1}(t)}{d t^{2}}+\frac{w_{1}}{Q_{1}} \frac{d y_{1}(t)}{d t}+G_{Q} \frac{d y_{1}(t)}{d t}+w_{1}^{2} y_{1}(t) \\
& =f_{T S}(t)+G_{\text {cont }}\left[y_{n}(t)\right] F_{1} \cos \left(w_{1} t\right) .
\end{aligned}
$$

$\frac{d^{2} y_{n}(t)}{d t^{2}}+\frac{w_{n}}{Q_{n}} \frac{d y_{n}(t)}{d t}+w_{n}^{2} y_{n}(t)=f_{T S}(t)+F_{n} \cos \left(w_{n} t\right)$

Selecting the range of $G_{\text {cont }}$ we can adjust the actuation range, e.g., if the free oscillation amplitude of $y_{1}(\mathrm{t})$ is $500 \mathrm{~nm}$ and $0.1 \leq$ $G_{\text {cont }} \leq 1$, the actuation range will be from $50 \mathrm{~nm}$ to $500 \mathrm{~nm}$, meaning that the height of the surface topography must be less than or equal to $450 \mathrm{~nm}$ in the scanned area.

\section{Simulation results}

The advantage of the proposed high-speed dynamic imaging method can be demonstrated theoretically or experimentally. However, a fast $\mathrm{X}-\mathrm{Y}$ scanner is required to demonstrate the full improvement in speed. In addition, the interaction force cannot be obtained experimentally in conventional AFM systems. Analytical approach is not suitable for comparison as well. Since we need to show how fast we can scan any given sample that can have a topography comparable to the amplitude of $y_{n}(t)$, the interaction force $f_{T S}(t)$ may not be linearized or may not even reach steadystate at the step edges of the sample topography. Therefore we need a simulation platform that allows a direct and accurate comparison of the proposed and the conventional imaging methods for the same tip-sample system.

Previously, we showed that the time-domain simulations of tipsample system can be performed using an electrical circuit simulator, and the obtained results has proven the reliability of simulations [34]. An important advantage is that the system electronics can easily be integrated into the simulations. The point-mass model equations for both fundamental and higher eigenmodes given in Eqs. (1) and (2) can be converted to the equivalent electrical circuits. To model the tipsample interactions, we use Derjaguin-Muller-Toporov contact model of a spherical tip [35]. We include the van der Waals forces to account for attractive surface forces. In the simulations, the parameters that we look for are the minimum time required to obtain a given sample topography with a certain accuracy and the applied peak transient forces. 
We assume that the operating environment is air. The fundamental eigenmode has a resonance frequency of $f_{1}=300 \mathrm{kHz}$, a spring constant of $k_{1}=10 \mathrm{~N} / \mathrm{m}$, and a $Q$-factor of $Q_{1}=100$. The higher eigenmode has a resonance frequency of $f=3 \mathrm{MHz}$, a spring constant of $k=100 \mathrm{~N} / \mathrm{m}$, and a $Q$-factor of $Q_{n}=100$. Note that for rectangular cantilever beams the higher eigenmode resonances and spring constants are at specific multiples of the fundamental one. In order not to restrict neither the analysis nor the cantilever type to a specific case, only the fundamental eigenmode parameters are chosen close to those of a typical tapping-mode cantilever. The tip-sample parameters are chosen to simulate a typical AFM experiment, where the tip radius is $R=10 \mathrm{~nm}$, the reduced tip-sample elasticity is $E_{r}=1 \mathrm{GPa}$, and the Hamaker constant is $A_{\mathrm{H}}=1 \times 10^{-19} \mathrm{~J}$.

To measure the higher eigenmode oscillation amplitude a peak detector [36] is employed. We choose the constant excitation mode of the FM-AFM [37], and to measure the higher eigenmode oscillation frequency an analog FM demodulator [38] is used. The outputs of peak detector and FM demodulator are connected to a PI controller. The output of the PI controller generates $G_{\text {cont }}\left[y_{n}(t)\right]$ to control the low-frequency actuation signal. The sum of lowfrequency actuation, high-frequency excitation, and $Q$-control signals drives the cantilever.

We first simulated the conventional tapping-mode experiment. In a typical AFM system, the Z-piezo has a resonance frequency of few $\mathrm{kHz}$, and in this simulation it is modeled as a second-order system with a resonance frequency of $3 \mathrm{kHz}$ and a $Q$-factor of 10 . We defined a sample surface with a $10 \mathrm{~nm}$ height as can be seen in Fig. 2. The PI controller is tuned by the Ziegler-Nichols method to achieve the minimum time required to obtain this surface feature with an acceptable error. The measurement error is calculated by

error $=\frac{\int \mid \text { sample surface }- \text { topography signal } \mid}{\int \mid \text { sample surface } \mid} \times 100 \%$.

In this study, we assumed that $30 \%$ measurement error is acceptable and the minimum time required is approximately $20 \mathrm{~ms}$ in this case. The second parameter that we are considering is the peak transient forces applied to the sample. In the conventional tapping-mode simulation, the free oscillation amplitude and the set-point values are $A_{1}=100 \mathrm{~nm}$ and $A_{\text {set }} / A_{1} \approx 70 \%$, respectively, and the peak forces are found to be about $60 \mathrm{nN}$.

Next, we simulated the proposed method. The free oscillation amplitude of the higher eigenmode and the set-point values are

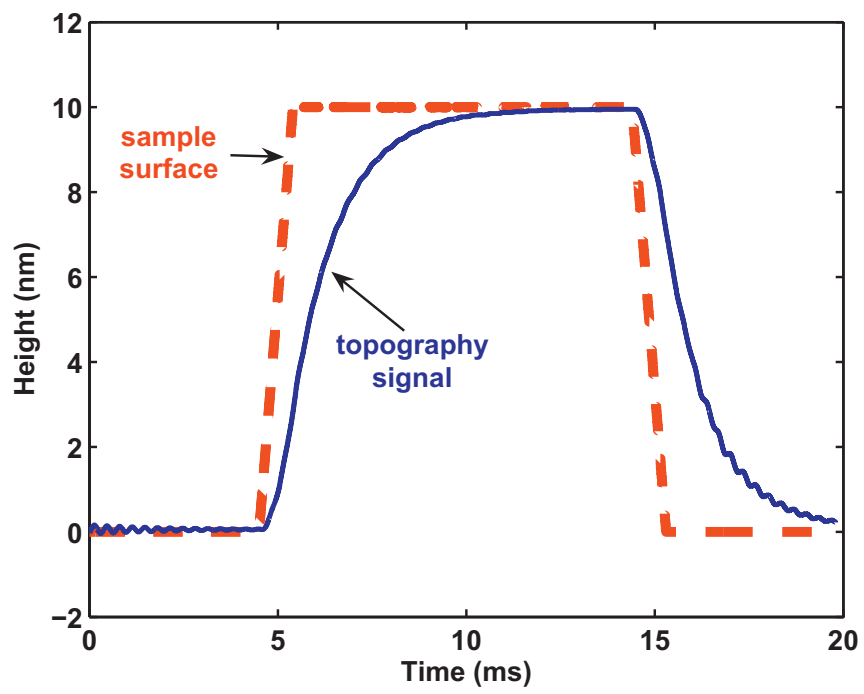

Fig. 2. The topography signal for the given sample surface in the conventional tapping-mode. chosen to be $A=12 \mathrm{~nm}$ and $A_{\text {set }} / A_{n} \approx 70 \%$ (for the AM-AFM system). In the FM-AFM system, the cantilever is kept oscillating at its higher eigenmode via positive feedback with a free oscillation amplitude of $A_{n}=12 \mathrm{~nm}$ and the set-point is chosen to be the $80 \%$ percent of the maximum value of the FM demodulator output. The actuation range parameter is chosen to be $0.5 \leq G_{\text {cont }} \leq 1$ and the free oscillation amplitude of the fundamental eigenmode is $A_{1}$ $=100 \mathrm{~nm}$, providing a $50 \mathrm{~nm}$ actuation range.

In Fig. 3, we see a section from the time-domain simulation of the proposed FM-AFM system where the peak transient forces reach their maximum of about $300 \mathrm{nN}$ due to an upward step in the surface topography. Note that this peak force occurs for only one cycle of fundamental oscillation, otherwise the peak forces are around $230 \mathrm{nN}$. Unlike the traditional FM-AFM imaging where the tip oscillates mostly in the attractive force region, we selected the set-point such that the tip also enters the repulsive force region as in the tapping-mode. The interaction force can have several repulsive peaks with smaller durations while the tip approaches the sample unlike the conventional tapping-mode. We can also clearly observe the $3 \mathrm{MHz}$ higher eigenmode oscillation superimposed on the $300 \mathrm{kHz}$ fundamental eigenmode oscillation. A similar interaction is also observed in the time-domain simulation of AM-AFM system, where the peak forces reach to approximately $210 \mathrm{nN}$.

Fig. 4 shows the variations of both the fundamental and the higher eigenmode oscillation amplitudes in the AM-AFM system as the tip scans a sample that has a $10 \mathrm{~nm}$ tall surface feature. When the tip passes the rising (or falling) edge of the surface topography, the higher eigenmode amplitude decreases (or increases). This variation in the higher eigenmode amplitude (the error signal) controls the fundamental eigenmode amplitude. The decreased fundamental eigenmode oscillation amplitude basically moves the tip away from the sample and hence performs the function of an actuator. We note that the fundamental amplitude is shifted down arbitrarily to be fitted into the figure. The result obtained from the FM-AFM simulation is given in Fig. 5, where the higher eigenmode oscillation frequency (the error signal) controls the fundamental eigenmode amplitude. In this figure, the curve denoted as higher eigenmode frequency is actually the FM demodulator output which decreases with increasing frequency. Hence as the tip passes the rising (or falling) edge of the surface topography, the higher eigenmode frequency increases (or decreases). By inverting the fundamental oscillation amplitude we can obtain the sample topography. If we assume that 30\% error, defined in Eq. (3), is acceptable, i.e., the topography signal adequately represents the sample surface, the minimum time required to obtain the defined surface feature is at least $360 \mu \mathrm{s}$ in the high-speed AM-AFM system. In the high-speed FM-AFM system, on the other hand, the minimum time required to obtain the same surface feature is at least $144 \mu$ s for the same error.

\section{Discussion}

If we compare the minimum required times to obtain the defined sample surface we see that the shortest time, i.e. the fastest speed, is achieved by the FM-AFM version of the proposed method. The ratio of the longest time duration, i.e. the slowest speed, obtained by the conventional tapping-mode to the time obtained by the FM-AFM version of the proposed method is $20 \mathrm{~ms} / 144 \mu \mathrm{s} \approx 140$. The ratio of the time obtained by the conventional tapping-mode to the time obtained by the AM-AFM version of the proposed method is $20 \mathrm{~ms} / 360 \mu \mathrm{s} \approx 55$. These results tell us that the imaging speed can be increased by about 2 orders of magnitude compared to conventional dynamic AFM imaging. It should be underlined that these results can be easily obtained even with an ordinary AFM cantilever. Another 


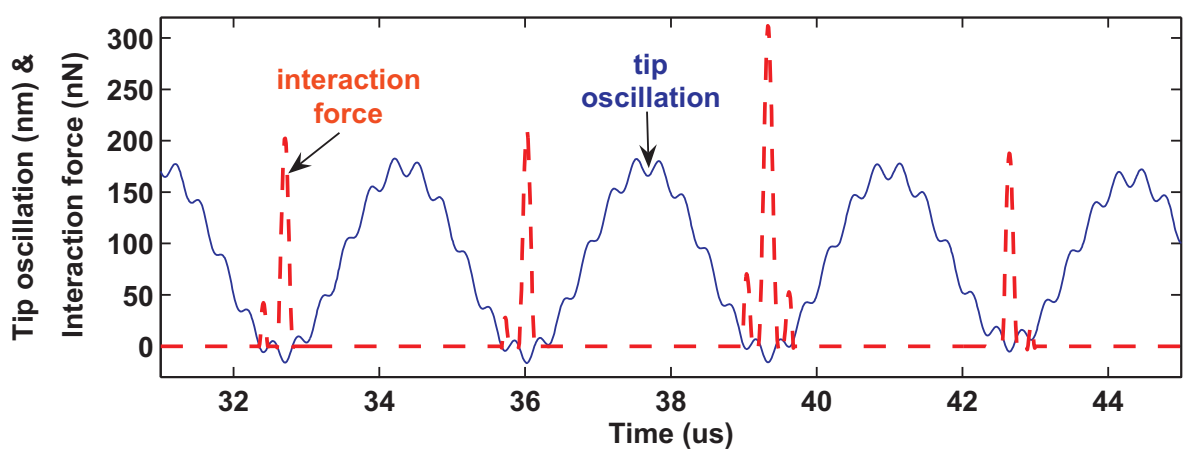

Fig. 3. The tip oscillation and the interaction force are plotted as a function of time in the proposed high-speed FM-AFM system.

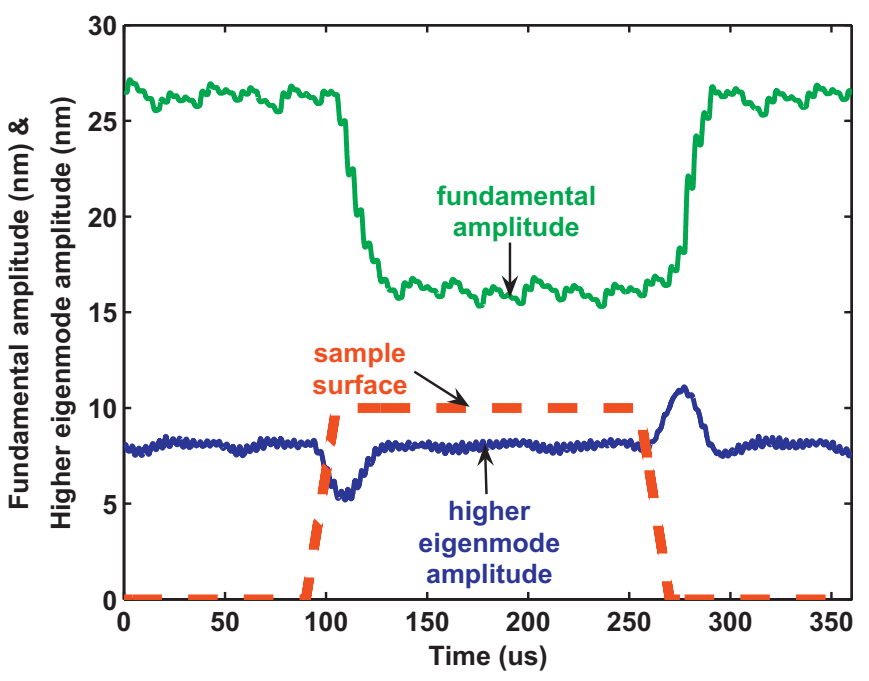

Fig. 4. Fundamental and higher eigenmode amplitudes as the tip experiences a surface feature of in the high-speed AM-AFM imaging system.

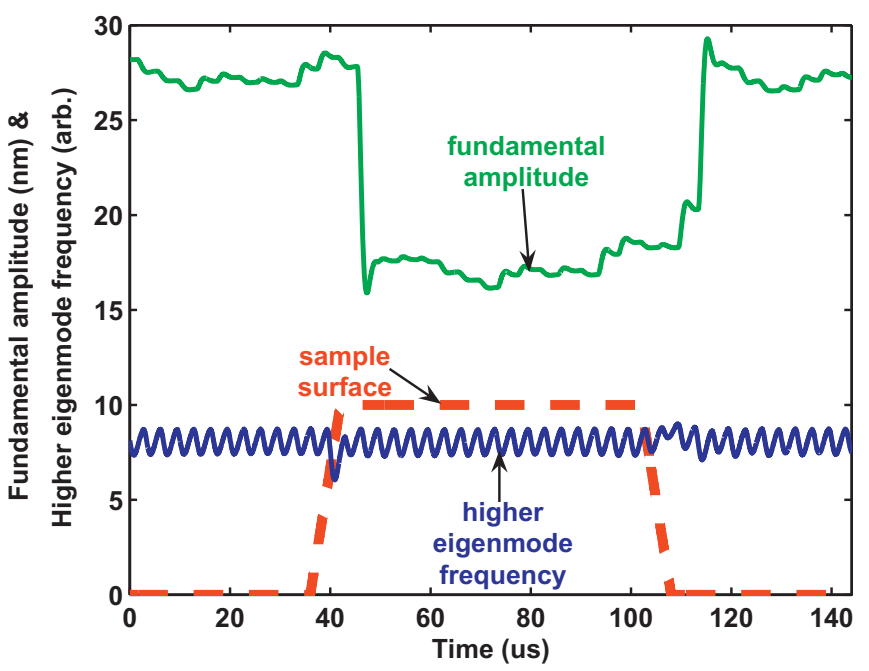

Fig. 5. Fundamental eigenmode amplitude and higher eigenmode frequency signals as the tip experiences a surface feature in the high-speed FM-AFM imaging system. The curve denoted as higher eigenmode frequency is actually the FM demodulator output which decreases with increasing frequency.

important point is that these results are obtained with a feedback controlled actuation of the $Q$-controlled fundamental eigenmode oscillation. It is not just recording the uncontrolled fundamental eigenmode oscillation amplitude, which would be both destructive (at the rising edges) and the tip can lose contact with the sample (at the falling edges).
The next question is how we can interpret these numbers, i.e., what is the meaning of minimum required time? If we perform an AFM experiment basically what we look for is that the time required to acquire one image (or frame). However, this time depends on many factors, namely, the pixel size, the area and roughness of the topography, the measurement accuracy, etc. To obtain speed in terms of image per second (or frame per second) we need to do some assumptions. Let us consider a sample that has a grating structure with a height of $10 \mathrm{~nm}$. Let us also assume that there are at most 5 of these surface features in the fast scan axis. Then, the acquisition of $100 \times 100$ pixel image of this sample will take $20 \mathrm{~ms} /$ featurex 5 features/scanx 100 scans/frame $=10 \mathrm{~s} /$ frame with the conventional tapping-mode imaging, assuming that the image is taken during trace or retrace only. Obviously when we increase the pixel size, or if there are more of these surface features which may or may not be periodic in the fast or slow scan directions, then the imaging time will increase. It means that with this conventional imaging setup the acquisition of one image will take from tens of seconds to few minutes, as usually practiced in classical tapping-mode experiments. On the other hand, the acquisition of one frame with the proposed method will be $144 \mu \mathrm{s} \times 5 \times 100=72 \mathrm{~ms}$ for the FM-AFM system, and $360 \mu \mathrm{s} \times$ $5 \times 100=180 \mathrm{~ms}$ for the AM-AFM system. In other words, $\approx 14 \mathrm{frames} / \mathrm{s}$ imaging speed for the FM-AFM system and $\approx 5$ frames/s imaging speed for the AM-AFM system is possible even with a regular AFM cantilever.

One other question is where the scanned area is taken into account in these calculations? The minimum required time may correspond to $1 \mathrm{~nm}, 1 \mu \mathrm{m}$, or any other value depending on the scanned area. Therefore the X-Y scanner must be capable of scanning each line with a speed of feature length/minimum required time. If, e.g., the scan size is $1 \mu \mathrm{m}$, then the minimum speed of $\mathrm{X}-\mathrm{Y}$ scanner in the fast scan axis must be $(1 \mu \mathrm{m} / 5) / 144 \mu \mathrm{s} \approx 1.4 \mathrm{~mm} / \mathrm{s}$.

We also investigated the effect of sample height on the measurement error and the peak transient forces. We used the same parameters and performed the simulations for a $2 \mathrm{~nm}$ and a $50 \mathrm{~nm}$ tall samples where the scan time is kept unchanged. In the simulations of a $50 \mathrm{~nm}$ tall sample, we chose $0.25 \leq G_{\text {cont }} \leq 1$ and $A_{1}$ $=150 \mathrm{~nm}$ to obtain an actuation range greater that the surface height. The measurement error is about 30\% for both AM-AFM and FM-AFM systems in the simulations of a $50 \mathrm{~nm}$ tall sample. On the other hand, the measurement error is found to be $54 \%$ for the AMAFM system and 72\% for the FM-AFM system in the simulations of a $2 \mathrm{~nm}$ tall sample due to an increasing effect of ripples observed in the topography signal. The maximum of transient forces occurring at the rising edges are decreased to $160 \mathrm{nN}$ (AM-AFM) and $240 \mathrm{nN}$ (FM-AFM) in the simulations of a $2 \mathrm{~nm}$ tall sample, whereas they are increased to $600 \mathrm{nN}$ (AM-AFM) and $760 \mathrm{nN}$ (FMAFM) in the simulations of a $50 \mathrm{~nm}$ tall sample.

To evaluate the effect of sample stiffness we performed simulations for two different samples $\left(E_{r}=0.1 \mathrm{GPa}\right.$ and $\left.E_{r}=10 \mathrm{GPa}\right)$, 
where the sample height is again $10 \mathrm{~nm}$. Keeping the scan time same, the error is found to be below 35\% for both samples, while the peak forces are about $60 \mathrm{nN}(0.1 \mathrm{GPa})$ and $400 \mathrm{nN}(10 \mathrm{GPa})$ in the AM-AFM system. The measurement error is found to be below $38 \%$ for both samples, while the peak forces are about $80 \mathrm{nN}$ $(0.1 \mathrm{GPa})$ and $600 \mathrm{nN}(10 \mathrm{GPa})$ in the FM-AFM system. In the simulations of a $0.1 \mathrm{GPa}$ sample, we increased the set-point slightly to decrease the indentation into the sample. In fact, selecting softer cantilevers for soft samples and choosing stiffer cantilevers for stiff samples is appropriate [39]. Hence we decreased the cantilever stiffness 10 times for both eigenmodes, while keeping the resonance frequencies and the quality factors unchanged, and performed simulations on the $1 \mathrm{GPa}$ sample. We found that the peak forces are reduced approximately to $40 \mathrm{nN}$ in the AM-AFM system and to $60 \mathrm{nN}$ in the FM-AFM system.

We observe that the fundamental and the higher eigenmode amplitudes are not smooth in the flat portions of the sample surface and the higher eigenmode frequency is oscillating around its set-point value (see Figs. 4 and 5). This is due to non-ideal filters, used in amplitude and frequency detection circuits, with which we cannot perfectly separate two frequency components of tip oscillation. Since we obtain the topography signal by inverting the fundamental oscillation amplitude, the ripples are seen in the topography signal as well. The magnitude of peak ripples are around $0.8 \mathrm{~nm}$ in the AM-AFM system and around $1 \mathrm{~nm}$ in the FMAFM system. These ripples cause large measurement error in low surface features as we have seen in the case of a $2 \mathrm{~nm}$ tall sample. We investigated the effect of fundamental and higher eigenmode resonance frequencies on these ripples and the measurement error. We both separated the fundamental and higher eigenmode resonances and increased the fundamental eigenmode resonance frequency such that $f_{1}=600 \mathrm{kHz}$ and $f_{n}=12 \mathrm{MHz}$. The topography signal is obtained by inverting the fundamental oscillation amplitude as shown in Fig. 6 for the AM-AFM system. In this case, the minimum time required to obtain the same surface feature is $180 \mu \mathrm{s}$ in the AM-AFM system and $84 \mu \mathrm{s}$ in the FM-AFM system (data not shown). Moreover the measurement errors are reduced to $20 \%$ and $27 \%$, and the peak ripples in the topography signals are decreased to about $0.5 \mathrm{~nm}$ and $0.7 \mathrm{~nm}$ for the AM-AFM and the FM-AFM systems, respectively. These results show that better imaging quality is achieved if the fundamental and higher eigenmode resonances are well separated, and the scan speed can be increased further by using cantilevers with higher resonance

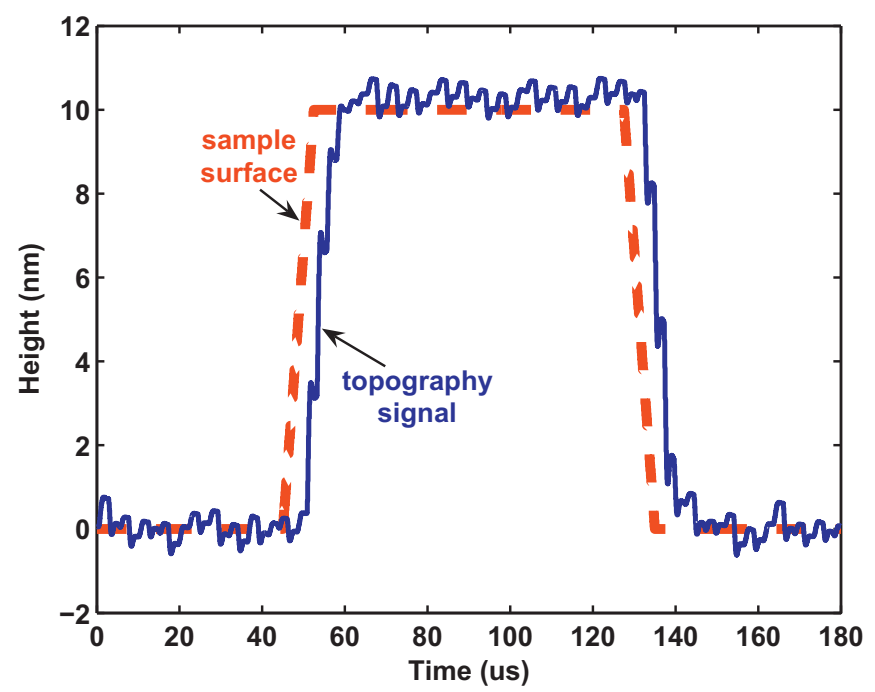

Fig. 6. The acquired topography signal in the high-speed AM-AFM imaging system. The fundamental and higher eigenmode resonance frequencies are $f_{1}=600 \mathrm{kHz}$ and $f_{n}=12 \mathrm{MHz}$. frequencies. Another point worth to mention is that the higher eigenmode resonance is not necessarily be an integer multiple of the fundamental resonance as might be inferred. We performed the simulations for $f_{1}=300 \mathrm{kHz}$ and $f_{n}=3.5 \mathrm{MHz}$ and obtained slightly better results.

Although the proposed system electronics is more complex compared to the conventional setups, it can be readily integrated into the existing AFM systems which employ regular cantilevers. The disadvantage coming at the expense of speed improvement is the increased peak forces due to stiffer higher eigenmode used for force sensing. Also, the higher eigenmode tip oscillation interacts for a very short time with the sample surface. When averaged with the oscillation cycles without tip-sample interaction, larger peak forces are created for the same set-point as compared to the regular tapping-mode imaging with higher eigenmode. We can reduce peak forces by using a softer cantilever. Another disadvantage is the smaller actuation range compared to conventional Z-piezo actuation. However, to achieve a quick actuation we need a small sized actuator. To balance the low-frequency topographic variations or a possible tilt on the sample surface, the Z-piezo of the conventional tapping-mode setups can be utilized.

By using special cantilevers having small spring constants and high resonance frequencies we can both increase the scan speed further and reduce the peak forces drastically. In fact, small cantilevers with spring constants less than $1 \mathrm{~N} / \mathrm{m}$ and resonance frequencies greater than $1 \mathrm{MHz}$ was employed in AFM systems with optical beam deflection detection $[7,13]$. The fastest speed achieved by using a cantilever with a spring constant of $0.2 \mathrm{~N} / \mathrm{m}$ and a resonance frequency of $1.2 \mathrm{MHz}$ is reported to be 25 frames/ $\mathrm{s}$ for a $200 \mathrm{~nm}$ scan size and $100 \times 100$ pixels, where the Z-actuator is reported to have a bandwidth of $370 \mathrm{kHz}$ and an actuation range of $1 \mu \mathrm{m}$ [13]. Our analysis have shown that for the same pixel size, the imaging speed of the proposed method in FM-AFM system will be approximately $(144 \mu \mathrm{s} / 84 \mu \mathrm{s}) \times 14$ frames/ $\mathrm{s} \approx 24 \mathrm{frames} / \mathrm{s}$ for a cantilever of $600 \mathrm{kHz}$ resonance frequency. Therefore we expect the imaging speed to be greater than 24 frames/s with the cantilever having $1.2 \mathrm{MHz}$ resonance frequency, and even more the peak transient forces will be much lower than those obtained in this study due to a very low spring constant of $0.2 \mathrm{~N} / \mathrm{m}$.

Finally, by integrating a fast X-Y scan unit to the system one can much effectively use the proposed method in high-speed dynamic AFM imaging. Recently developed X-Y scanners $[15,18]$ are capable of scanning at a rate greater than $1.4 \mathrm{~mm} / \mathrm{s}$ in the fast scan axis. In the former study, contact-mode images at a rate of 70 frames/s was acquired without electronic feedback [15], whereas in the latter one, a scan velocity of $8.8 \mathrm{~mm} / \mathrm{s}$ of and imaging rate of 46 frames/s was achieved again in contact-mode with feedback, where the closed-loop bandwidth of the $\mathrm{Z}$ actuator was reported to be the limiting factor [18].

\section{Conclusion}

High-speed imaging and characterization of nanoscale structures, devices, and systems has become increasingly important as stated in the National Nanotechnology Initiative report. In this work, we presented a high-speed and dynamic actuation method with feedback that uses an ordinary cantilever both as the sensor and the actuator. The method eliminates the shortcoming of the bulky Z-piezo by utilizing $Q$-controlled fundamental eigenmode oscillation as an actuator. We performed the time-domain simulations of the proposed method and the conventional method on the same sample having sharp edges. This allowed us to evaluate the performance of the proposed method and directly compare it to the conventional imaging method which may not be easily done 
either analytically or experimentally. We showed that the imaging speed can be improved by about 2 orders of magnitude compared to conventional dynamic AFM imaging. We investigated the method in terms of scan time, peak transient forces applied to the sample, and the measurement error for different sample and cantilever parameters. We have shown that the imaging speed can be increased further by using cantilevers with higher resonance frequencies. We have already implemented the proposed highspeed method and performed the proof-of-principle experiments on a commercial AFM system [40]. This method will indeed minimize the time spent for dynamic AFM imaging experiments without requiring special or small cantilevers. It can also enable the dynamic AFM systems to be used widely in industrial scale nano-metrology and manipulation.

\section{Acknowledgement}

The author thanks funding by TUBITAK (Grant no. 110T732).

\section{References}

[1] G. Binnig, C.F. Quate, Ch Gerber, Phys. Rev. Lett. 56 (1986) 930.

[2] G. Schitter, P. Menold, H.F. Knapp, F. Allgower, A. Stemmer, Rev. Sci. Instrum 72 (2001) 3320.

[3] G. Schitter, F. Allgower, A. Stemmer, Nanotechnology 15 (2004) 108.

[4] G.E. Fantner, P. Hegarty, J.H. Kindt, G. Schitter, G.A.G. Cidade, P.K. Hansma, Rev. Sci. Instrum. 76 (2005) 026118.

[5] T. Uchihashi, T. Ando, H. Yamashita, Appl. Phys. Lett. 89 (2006) 213112.

[6] D. Lee, H. Lee, N.S. Lee, K.B. Kim, Y. Seo, Curr. Appl. Phys. 12 (2012) 989.

[7] G.E. Fantner, G. Schitter, J.H. Kindt, T. Ivanov, K. Ivanova, R. Patel, N. HoltenAndersen, J. Adams, P.J. Thurner, I.W. Rangelow, P.K. Hansma, Ultramicroscopy 106 (2006) 881.

[8] T. Ando, T. Uchihashi, T. Fukuma, Prog. Surf. Sci. 83 (2008) 337.

[9] D.A. Walters, J.P. Cleveland, N.H. Thomson, P.K. Hansma, M.A. Wendman, G. Gurley, V. Elings, Rev. Sci. Instrum 67 (1996) 3583.

[10] T.E. Schaffer, P.K. Hansma, J. Appl. Phys. 84 (1998) 4661.

[11] M.B. Viani, T.E. Schaffer, G.T. Paloczi, L.I. Pietrasanta, B.L. Smith, J.B. Thompson, M. Richter, M. Rief, H.E. Gaub, K.W. Plaxco, A.N. Cleland, H.G. Hansma, P. K. Hansma, Rev. Sci. Instrum. 70 (1999) 4300.

[12] N. Kodera, D. Yamamoto, R. Ishikawa, T. Ando, Nature 468 (2010) 72.
[13] T. Ando, Nanotechnology 23 (2012) 062001.

[14] J.H. Kindt, G.E. Fantner, J.A. Cutroni, P.K. Hansma, Ultramicroscopy 100 (2004) 259.

[15] A.D.L. Humphris, M.J. Miles, J.K. Hobbs, Appl. Phys. Lett. 86 (2005) 034106.

[16] T. Fukuma, Y. Okazaki, N. Kodera, T. Uchihashi, T. Ando, Appl. Phys. Lett. 92 (2008) 243119.

[17] B. Zhao, J.P. Howard-Knight, A.D.L. Humphris, L. Kailas, E.C. Ratcliffe, S.J. Foster, J.K. Hobbs, Rev. Sci. Instrum. 80 (2009) 093707.

[18] C. Braunsmann, T.E. Schäffer, Nanotechnology 21 (2010) 225705.

[19] S.R. Manalis, S.C. Minne, C.F. Quate, Appl. Phys. Lett. 68 (1996) 871.

[20] T. Sulchek, S.C. Minne, J.D. Adams, D.A. Fletcher, A. Atalar, C.F. Quate, D. M. Adderton, Appl. Phys. Lett. 75 (1999) 1637.

[21] T. Sulchek, R. Hsieh, J.D. Adams, S.C. Minne, C.F. Quate, D.M. Adderton, Rev. Sci. Instrum. 71 (2000) 2097.

[22] T. Akiyama, U. Staufer, N.F. de Rooij, Rev. Sci. Instrum. 73 (2002) 2643.

[23] B. Rogers, L. Manning, T. Sulchek, J.D. Adams, Ultramicroscopy 100 (2004) 267.

[24] F.L. Degertekin, A.G. Onaran, M. Balantekin, W. Lee, N.A. Hall, C.F. Quate, Appl. Phys. Lett. 87 (2005) 213109.

[25] A.G. Onaran, M. Balantekin, W. Lee, W.L. Hughes, B.A. Buchine, R.O. Guldiken, Z. Parlak, C.F. Quate, F.L. Degertekin, Rev. Sci. Instrum. 77 (2006) 023501.

[26] L.M. Picco, L. Bozec, A. Ulcinas, D.J. Engledew, M. Antognozzi, M.A. Horton, M. J. Miles, Nanotechnology 18 (2007) 044030.

[27] L.M. Picco, P.G. Dunton, A. Ulcinas, D.J. Engledew, O. Hoshi, T. Ushiki, M.J. Miles, Nanotechnology 19 (2008) 384018.

[28] R. Garcia, R. Perez, Surf. Sci. Rep. 47 (2002) 197.

[29] G. Chawla, S.D. Solares, Meas. Sci. Technol. 20 (2009) 015501.

[30] Y. Naitoh, Z. Ma, Y.J. Li, M. Kageshima, Y. Sugawara, J. Vac. Sci. Technol. B 28 (2010) 1210

[31] M.D. Aksoy, A. Atalar, Phys. Rev. B 83 (2011) 075416.

[32] T. Sulchek, R. Hsieh, J.D. Adams, G.G. Yaralioglu, S.C. Minne, C.F. Quate, J. P. Cleveland, A. Atalar, D.M. Adderton, Appl. Phys. Lett. 76 (2000) 1473.

[33] M. Antognozzi, M.D. Szczelkun, A.D.L. Humphris, M.J. Miles, Appl. Phys. Lett. 82 (2003) 2761.

[34] M. Balantekin, A. Atalar, Appl. Surf. Sci. 205 (2003) 86.

[35] B. Bhushan, Handbook of Micro/Nanotribology, CRC, Boca Raton, Florida, 1999.

[36] M. Balantekin, F.L. Degertekin, Ultramicroscopy 111 (2011) 1388.

[37] H. Ueyama, Y. Sugawara, S. Morita, Appl. Phys. A: Mater. Sci. Process. 66 (1998) S295.

[38] T.R. Albrecht, P. Grutter, D. Horne, D. Rugar, J. Appl. Phys. 69 (1991) 668.

[39] M. Balantekin, A. Atalar, Phys. Rev. B 71 (2005) 125416.

[40] M. Balantekin, S. Satır, D. Torello, F.L. Değertekin, 2014 (submitted for publication). 